\title{
Phraseologie in arealen Bezügen: ein Problemaufriss
}

\author{
Elisabeth Piirainen (Steinfurt/Westfalen)
}

\begin{abstract}
Traditional phraseology research tends to start from the assumption that the geographical spread of idioms of one individual language is identical to the area where that individual language is spoken. This assumption, however, is not correct for all idioms. Especially with respect to German, one has to take into account that many idioms are known only within a limited area, e. g. because of their dialectal origins. Linguistic geographical studies on phraseology are usually restricted to aspects of the German pluricentricity. What has been overlooked is the fact that an independent phraseology was beginning to develop in the former GDR. Furthermore, the fact that idioms may exist in much larger areas than that of one individual language is largely unexplored. The aim of this paper is to point to problems of phraseology research in an areal framework. Two case studies on the distribution of idioms demonstrate that any research in areal phraseology must start with comprehensive empirical work.
\end{abstract}

\footnotetext{
Zwei griechische Gelehrte führten einen Disput über die Anzahl der Zähne im Maul des Pferdes. Durch gründliches Philosophieren war der eine zum Ergebnis von 64 gelangt, während der andere, seiner Eingebung folgend, auf 48 beharrte. Hinzu trat Empirokles, öffnete das Maul eines Gaules und sprach: "Beim Zeus, so schauet doch!"
}

\section{$1 \quad$ Vorbemerkung}

Raum und Zeit haben als die beiden Invariablen zu gelten, derer sich Humanwissenschaften kaum gänzlich entziehen können. Für die Phraseologieforschung gilt jedoch, dass der zeitlichen Dimension weitaus mehr Beachtung geschenkt wird als der räumlichen. In zuverlässigen Darstellungen (theoretischer oder praktischer, z. B. phraseographischer Art) wird der diachronische Aspekt berücksichtigt, indem das Veralten oder in Mode kommen eines Phraseologismus dokumentiert wird (z. B. "antiquiert", "Neologismus"); diatopische Markierungen finden sich hingegen zumeist nicht. Phraseologieforschung in historischen Bezügen wird größeres Interesse beigemessen denn jener in arealen Bezügen. Bis vor kurzem fehlte ein Bewusstsein dafür, dass Phraseologismen nur in einem begrenzten Areal Gültigkeit haben könnten. Die traditionelle Phraseologieforschung geht von der Vorstellung eines homogenen Bestandes an Phraseologismen, oberhalb einer regionalen Ebene, d. h. von einer Gleichsetzung von (National-)Sprache und Raum (in dem diese Sprache gesprochen wird) 
aus. Gerade für eine Sprache wie Deutsch, das historisch auf einer Reihe von Dialekten basiert, ist dieses Konzept nicht haltbar. Die Dialekte haben deutliche Spuren in der Phraseologie sowohl der Schriftsprache als auch der standardnahen, mündlich gebrauchten Umgangssprachen hinterlassen. Andererseits wird beobachtet, dass bestimmte Phraseologismen in weit größeren Sprachräumen als dem einer Einzelsprache existieren. Phraseologieforschung in europäischen Bezügen und darüber hinaus steht ebenfalls noch am Anfang und verlangt nach sorgfältigen empirischen Erhebungen. Ziel dieses Beitrags ist es, auf die Problemkomplexe und Forschungslücken zunächst einmal hinzuweisen. Im Anhang werden zwei Projekte und einzelne Lösungsmöglichkeiten vorgestellt. Das Verhältnis der Phraseologie zum Raum wird aufgrund der folgenden Verbreitungstypen betrachtet, von der kleinsten Einheit fortschreitend zu stets größeren Arealen:

(i) dialektale Phraseologismen

(ii) regional verbreitete Phraseologismen der Umgangssprachen

(iii) innerhalb eines Staatsgebietes gültige Phraseologismen

(iv) interlingual weit verbreitete Phraseologismen

\section{$2 \quad$ Arealphraseologie: zum Stand der Forschung}

Arealphraseologie wird hier in Anlehnung an den Terminus Areallinguistik gebraucht, wie er von Goossens (1980: 454) definiert wird, d. h. als sprachgeographische Disziplin. ${ }^{1}$ Wie die oben genannten Verbreitungstypen zeigen, wird Arealphraseologie jedoch nicht nur auf dialektologische, sondern auch auf weitere mit der Phraseologie verbundene sprachräumliche Fragestellungen bezogen. In der phraseologischen Literatur begegnet vereinzelt der Terminus areale Phraseologie, in wieder anderem Sinne, als Teilbereich der kontrastiven Phraseologie, z. B. beim Vergleich von Kroatisch und Deutsch mit dem Russischen bei Matešić/Petermann (1987).

In diesem Beitrag sind folgende Eingrenzungen der Thematik vorgesehen: Obwohl sich die Phraseologie durch ihre interdisziplinäre Herangehensweisen auszeichnet, werden hier vor allem germanistische Themen erörtert (abgesehen naturgemäß von Punkt (iv)), weil mir für andere Sprachen, auch für phraseologisch umfassend untersuchte Standardsprachen wie Russisch, Französisch oder Ungarisch, keine Studien zu arealen Phänomenen bekannt sind.

Zum anderen ist eine Beschränkung auf die in der phraseologischen Literatur zumeist als zentral angesehene phraseologische Klasse der Idiome erforderlich, da für andere Klassen kaum Untersuchungen existieren. Zwar hat die Parömiologie von den frühesten Zeiten an geolinguistische Aspekte berücksichtigt, sei es bei Erhebungen dialektaler oder interlingual verbreiteter Sprichwörter, doch sind jene Studien nicht vorrangig in der Linguistik, sondern in

\footnotetext{
${ }^{1}$ Zur Geschichte und zu anderen Verwendungsweisen des Terminus Areallinguistik cf. Sternmann/Gutschmidt (1989: 271ff).
} 
ethnologischen und kulturwissenschaftlichen Disziplinen angesiedelt. Für Fragen nach ihrer arealen Geltung sind weitere phraseologische Gruppen, etwa die schwach idiomatischen grammatischen (wie dem auch sei) und onymischen Phraseologismen (Das Rote Kreuz) oder Funktionsverbgefüge (etw. gelangt zur Anwendung) vermutlich weniger aufschlussreich. $\mathrm{Ob}$ Kollokationen wie sich die Zähne putzen von arealen Phänomenen betroffen sein können, ist nicht bekannt; es fehlt an Voruntersuchungen jeglicher Art.

In deutschen Sprachatlaswerken ${ }^{2}$ werden vereinzelt kommunikative Routineformeln berücksichtigt, da sie für die raumstrukturierende Funktion bekannt sind. Der "Wortatlas der deutschen Umgangssprachen" (Eichhoff 1977ff: 1, 47) enthält die Karte "Gruß beim Betreten eines Geschäfts (am Nachmittag)". Erkennbar sind die beiden großen Sprachräume Guten Tag nördlich und Grüß Gott südlich der Mainlinie, am Rande außerdem Grüezi, grüeß ech (Schweiz) und Moin (in Teilen Nordwestdeutschlands). Nur einige weitere, am Rande der Phraseologie angesiedelte feste Wortverbindungen sind in Sprachatlanten gedrungen. Es sind z. B. Bezeichnungen von Kinderspielen (Purzelbaum schlagen; Himmel und Hölle; jemanden Huckepack nehmen), die Eingang in den Wortatlas (Eichhoff 1977ff: 4, 24ff) oder den "Sprachatlas der deutschen Schweiz" (cf. Zürrer im Druck) gefunden haben und klare Verbreitungsareale erkennen lassen. Der "Atlas zur deutschen Alltagssprache" (Elspaß/Möller 2003ff) enthält unter zahlreichen Einzelwörtern bis jetzt nur eine feste Wortfügung: eins gemerkt vs. eins im Sinn (beim Rechnen).

Die diatopische Gliederung des deutschen Sprachraums ist seit langem gut erforscht, vgl. zur Laut- und Formenlehre Wenkers Fragesatz-Erhebungen seit 1876 (Wenker 1881) sowie zur Lexik Kretschmers "Wortgeographie der hochdeutschen Umgangssprache" (1918) oder die Arbeiten zum Deutschen Wortatlas (DWA) seit 1939 (Mitzka et al. 1951ff). Diesen umfassenden arealen Studien steht seitens der Phraseologie nichts Vergleichbares gegenüber. Als einzige groß angelegte Arbeit zur Arealphraseologie ist Grober-Glück (1974) zu nennen, die auf Erhebungen zum "Atlas der deutschen Volkskunde" in den 30er Jahren zurückführt und die Verbreitung bestimmter Umgangsformeln, Brauchtumsstereotype und fester Vergleiche im damaligen deutschen Sprachraum behandelt. ${ }^{3}$ Im Folgenden werden die vier Verbreitungstypen (auch im Hinblick auf den "Stand der Forschung") ${ }^{4}$ getrennt betrachtet (Abschnitte 3-6). Am Ende des Beitrags werden zwei Projekte vorgestellt: zur Erforschung von Idiomen des Verbreitungstyps (ii) im Anhang Teil I sowie des Typs (iv) im Anhang Teil II).

\section{Dialektologie und Phraseologie}

Die Anfänge der Dialektologie als sprachwissenschaftliche Disziplin reichen in die Mitte des 19. Jahrhunderts zurück, als in Westeuropa Wörterbücher und Grammatiken von

\footnotetext{
${ }^{2}$ Die bedeutsame Rolle der Gruß- und Höflichkeitsformeln des Japanischen hat in einem Atlaswerk seinen Niederschlag gefunden, cf. Ebata (2003).

${ }^{3}$ Die Arbeit ist im Rahmen der deutschen Volkskunde entstanden. Aus sprachwissenschaftlicher Sicht wurde z. T. die nicht eindeutige Trennung zwischen Dialekten und hochdeutschen Umgangssprachen kritisiert.

${ }^{4}$ Vgl. auch die Übersicht in Filatkina (2005: 23ff); Piirainen (2000: 24ff, im Druck a).
} 
Ortsdialekten entstanden. Einen Hauptgegenstand bildet sodann die Beziehung linguistischer Daten zum Raum, d. h. die Ermittlung von Sprachräumen aufgrund von Merkmalen, die sich in (Bündeln von) Isoglossen auf Atlaskarten abbilden lassen. Empirisch basierte Theoriebildung sowie ein Theoriebewusstsein hinsichtlich des raumbildenden Potenzials sprachlicher Faktoren sind der Dialektgeographie zu verdanken, von deren Methodenspektrum die Phraseologieforschung profitieren könnte. Andererseits verhält sich die traditionsreiche deutsche Dialektologie eher reserviert gegenüber neu in die Linguistik vordringende Disziplinen. Abraham/Beyer (1993: 7) schildern die damals schroffe Ablehnung der Forschungsrichtung "Dialektsyntax", wobei dieser Widerstand inzwischen überwunden ist. "Dialektphraseologie" bildet jedoch weiterhin kein Forschungsthema der Dialektologie. 5

Besser ist es in dieser Hinsicht um die gegenwärtige Phraseologieforschung bestellt, da Studien zur Dialektphraseologie mittlerweile in das Forschungsspektrum einbezogen werden: In dem geplanten Internationalen Handbuch zur Phraseologie (HSK, Burger et al. im Druck) wird es mehrere Artikel zur Dialektphraseologie geben; auf den Tagungen der Europäischen Gesellschaft für Phraseologie (http://www.europhras.org) sind Beiträge zu dialektalen Themen vertreten. Bisher liegen zwei Monographien vor, die - auf umfassenden Materialerhebungen, direkten und indirekten Befragungen basierend - dialektale Phraseologie jeweils einer fest umrissenen Region systematisch und im Rahmen linguistischer Theorien untersuchen: zu einem inzwischen fast ausgestorbenen niederdeutschen Basisdialekt, dem "Westmünsterländischen" im westlichen Westfalen (Piirainen 2000), sowie zum Lëtzebuergeschen, der recht vitalen Nationalsprache Luxemburgs, die historisch auf westmoselfränkische Mundarten zurückgeht (Filatkina 2005). Einige weitere Untersuchungen sind in Arbeit: zur Phraseologie schweizerdeutscher und alpiner Dialekte cf. u. a. Burger (2002); Zürrer/Burger (2004) und zur Phraseologie des Pfälzischen, Knop (in Vorbereitung).

Die genannten Arbeiten haben eine Reihe von Ergebnissen erbracht. So unterliegen Dialektphraseologismen zwar einer inneren Norm, doch verlangt die Beschreibung jener Norm nach einem flexibleren Umgang, da eine größere morphosyntaktische und lexikalische Variabilität zu verzeichnen ist. Ferner zeigen die untersuchten Dialektphraseologien eine gegenüber den Standardsprachen kulturelle Eigenständigkeit, die sich in Metaphern und Symbolen ebenso wie in gesamten Ausgangskonzepten (Realienwelt der betreffenden Dialektgemeinschaften) und semantischen Feldern (z. B. Salienz einzelner Zielkonzepte aufgrund bestimmter Wertvorstellungen) manifestieren kann. Schließlich sind Unterschiede der pragmatischen Funktionen zu nennen, die von einer geringeren Breite der stilistischen Register bis zu stärker ausgeprägten geschlechtsspezifischen Restriktionen und euphemistischen Umschreibungen reichen.

\footnotetext{
5 Auch in jüngeren Einführungen zur Dialektologie (z. B. Löffler 2003; Niebaum/Macha 2006) wird Dialektphraseologie nicht behandelt. Das Gleiche gilt für internationale Kompendien, z. B. Chambers/Trudgill (2002), und Dialektologie-Tagungen: Sektionen zur Phraseologie finden sich weder bei den internationalen Konferenzen "Methods in Dialectology" (2002 in Joensuu, 2005 in Moncton/Kanada) noch bei Tagungen der IGDD (der Internationalen Gesellschaft für deutsche Dialektologie, 2003 in Marburg, 2006 in Wien) (cf. Filppula 2005; Eggers et al. 2005).
} 
Diese Ergebnisse beruhen auf dem Kontrast zwischen Dialekt und Standardsprachen; sie berühren den Hauptgegenstand dieses Beitrags, die Beziehung der Phraseologie zum Raum, nur bedingt. Hingegen betrifft ein laufendes viel versprechendes Projekt über "Moselfränkisch diesseits und jenseits der Staatsgrenze" (Filatkina in Vorbereitung) eben dieses Thema. Die Erhebungen zeigen, dass die Staatsgrenze die Phraseologie des Westmoselfränkischen deutlich in zwei Gebiete teilt, sich jedoch nur teilweise zu einer Verständnishürde entwickelt: Die Idiome werden auf der jeweils anderen Seite der Grenze zwar verstanden, aber entweder nicht oder in einer anderen Form gebraucht. Dies ist ein innovativer Ansatz innerhalb der Dialekt- und Arealphraseologie. Zur Funktion des Staatsgebietes als Differenzierungsfaktor cf. auch Abschnitt 5.

\section{Phraseologie und regionale Umgangssprachen}

Untersuchungen zur begrenzten räumlichen Gültigkeit von Idiomen des Hochdeutschen bzw. der (standardnahen oder nichtstandardlichen) deutschen Umgangssprachen gehen entweder vom einzelnen Idiom aus oder von der Region selbst, deren sprachliche Sonderstellung bereits aus anderen Zusammenhängen bekannt sein mag. Arbeiten des zweiten Typs sind äußerst selten. Hier ist die Einzelstudie von Crede/Lakemper (1998) zur Phraseologie des Ruhrgebiets zu nennen. Anhand von Fragebögen werden zunächst die 20 bekanntesten ruhrgebietstypischen Idiome ermittelt, ${ }^{6}$ diese sodann mit Hilfe eines Geo-Informationssystems auf Karten projiziert. In dieser Arbeit kann die postulierte sprachliche Homogenität des Ruhrgebiets widerlegt und die Tendenz zu einer ost-westlichen Zweiteilung aufgezeigt werden.

Andere Studien zur Arealphraseologie innerhalb der deutschen Umgangssprachen nehmen die Idiome selbst als Ausgangspunkt. Im Anhang Teil I wird auf ein Projekt näher eingegangen, das eine genauere Lokalisierung von Idiomen im Raum der Bundesrepublik zum Ziel hat. Ausgangspunkt bildeten zunächst Idiome, die in Wörterbüchern diatopisch markiert sind. Es zeigte sich, dass Angaben wie bayr., berlin., landsch., nordd. im damaligen Duden 11 (1998), ${ }^{7}$ über deren Quellen oder empirische Grundlagen nichts mitgeteilt wird, mehrheitlich der Sprachrealität nicht entsprechen. So ist das als berlinisch markierte Idiom es regnet Schusterjungen in Berlin nicht (mehr) bekannt, eher im süddeutschen Raum, dort jedoch in der Form es regnet Schusterbuben (Piirainen 2002a: 38).

Die Phraseologieforschung in der DDR hat sich mit der diatopischen Markierung von Idiomen selbst nicht befasst. Dennoch können einige Publikationen Leipziger Provenienz

\footnotetext{
6 Es ist bei derartigen Untersuchungen vermutlich nie auszuschließen, dass regionaltypische Idiome auch außerhalb der betreffenden Region bekannt sind. Einige der angeführten Ruhrgebiets-Idiome führen auf das Niederdeutsche zurück und sind deshalb auch in der nördlich angrenzenden Umgangssprache des Münsterlandes anzutreffen (auf'n Stupp, das arme Tier kriegen), andere wurden vom Ruhrgebiet ausgehend auch in größeren Sprachräumen populär (über die Wupper gehen, Schicht im Schacht).

${ }^{7}$ Mitglieder der Dudenredaktion haben sich an dem im Anhang Teil I zu schildernden Umfrageprojekt tatkräftig beteiligt. Zwar lagen für die Neuauflage (Duden 11 2002) noch keine Einzelergebnisse des Projekts vor, doch konnten in der 2. Auflage bereits viele Verbesserungen der diatopischen Angaben vorgenommen werden.
} 
indirekt als Materialquelle genutzt werden, da dort verschiedene Idiome auftauchen, die in Westdeutschland völlig unbekannt waren, ${ }^{8}$ wobei die Autoren die Idiome für im ganzen deutschen Sprachgebiet gültig gehalten haben müssen. Es geht hier nicht um Kritik an diesen Arbeiten, sondern darum zu zeigen, dass jegliches Bewusstsein für die Arealität in der Einschätzung von Idiomen fehlte. Man ging davon aus, dass die eigene phraseologische Kompetenz der intersubjektiv standardsprachlichen entsprach. Es zeigte sich, dass es sich hier nicht nur um regional- bzw. DDR-typische Idiome, sondern um unmittelbare Übernahme aus den thüringisch-obersächsischen Mundarten handelt (Piirainen 2003a).

Dem kontaktlinguistischen Phänomen der Transferenz von Phraseologismen der bodenständigen Dialekte in die Umgangssprachen wurde bisher zu wenig Aufmerksamkeit gewidmet. Allein für die westfälisch geprägte Umgangssprache des Münsterlandes könnte, aufgrund teilnehmender Beobachtung, eine Fülle von in Umlauf befindlichen Idiomen genannt werden, die sich als Übersetzungstransfer aus dem einst dort gesprochenen Niederdeutschen erweisen. Usualisiert sind Wendungen wie ein Auto/... in'n Tott fahren ('Totalschaden verursachen'), etwas noch vor der Brust haben ('etwas noch vor sich haben'), das ist doch mehr als (wie) ins/im Näpfchen geht! (Ausdruck der Empörung), sie kommt nicht aus den Sträuchern/aus die Sträucher ('sie kommt bei der Arbeit nicht voran') oder er sitzt beim Pastor auf den Knien/auf die Knie ('er ist scheinheilig fromm'). ${ }^{9}$ Diese Idiome wurden in ihrer hochdeutschen Form bisher nicht lexikographisch erfasst. Gelegentlich sind sie in der lokalen Tagespresse zu finden (am ehesten bei der Wiedergabe mündlicher Äußerungen); einer Textkorpora- oder Internetrecherche würden sie sich jedoch entziehen.

\section{Phraseologie plurizentrischer Sprachen}

Der australische Germanist österreichischer Abstammung M. Clyne stellte 1984, in Analogie zu den unterschiedlichen Varietäten des weltweit verbreiteten Englisch, ${ }^{10}$ auch für die deutsche Sprache ein "plurizentrisches" Modell vor, das von Sprachwissenschaftlern nicht nur der damaligen DDR, sondern auch der anderen deutschsprachigen Nationen mit Interesse aufgenommen und diskutiert wurde. Hier sollen nicht die weitreichenden sprachpolitischen

\footnotetext{
${ }^{8}$ Es sind Idiome wie Fettlebe machen, Habchen und Babchen, Schliff backen (Fleischer 1982: 50ff), den Hut (für etwas) aufhaben, nicht aus der Knete/Asche kommen, nicht ganz ausgeklebt sein, einen Aufriss machen, fertig auf dem Docht sein, etwas unter Ulk verbuchen (Fleischer 1987: 310f.) oder sich an etwas ein Gütchen tun, sich ein Bewerbchen machen, bei jmdm. abgegessen haben (Palm 1995: 30, 52).

9 Die eindeutige Zuordnung derartiger Ausdrücke zum Niederdeutschen ist anhand der Idiomsammlungen (Piirainen 2000, Teil 2) möglich. Vgl. weitere Beispiele in Piirainen (1999, 2004b). Der umgekehrte Prozess ist ebenfalls zu beobachten, wenn Idiome der überdachenden Standardsprache wie Fremdwörter oder Kalkierungen in den Basisdialekt vordringen (z. B. Piirainen 2004a: 50ff).

10 Der amerikanische Phraseologieforscher A. Makkai geht bereits 1972 auf den Plurizentrismus ein; der Terminus für 'Varietät' lautet im Englischen jedoch dialect. Wenn Makkai (1972: 18) die Kategorie "idioms restricted to one or several dialects" einführt, so referiert er damit auf die Standardvarietäten des Englischen in England, Kanada, Australien, Neuseeland, USA Ostküste, USA Westküste, Midwest USA usw. Für andere plurizentrische Sprachen ist dieses Problem ebenfalls bekannt, z. B. für Portugiesisch in Brasilien. Zum QuebecFranzösischen wird ein phraseologisches Spezialwörterbuch erstellt (Dawes 2003); zahlreiche Idiome finden sich bereits in dem Allgemeinwörterbuch von Meney (1999).
} 
Aspekte dieses Modells, sondern allein die Auswirkungen auf die Phraseologieforschung erörtert werden. So beschränkte sich die Einbeziehung der Diatopik in phraseologische Untersuchungen des Deutschen lange Zeit hindurch fast ganz auf den Aspekt der "Plurizentrik" sensu Clyne, d.h. auf das Vorkommen von Idiomen in den nationalen Varietäten Österreichisches Deutsch, Schweizerhochdeutsch und Deutsch in der Bundesrepublik (cf. Burger 2003: 195ff). Angesichts der Seltenheit dialektal- und arealphraseologischer Studien sind die nationalen Varietäten sogar überdurchschnittlich oft Gegenstand der Phraseologieforschung gewesen, zum Schweizerhochdeutschen z. B. Burger (1995, 1996, 1998), zum Österreichischen z. B. Eismann (1991), Földes (1992, 1996: 36ff). Dennoch wird das Fehlen zuverlässiger Differenzwörterbücher oft hervorgehoben (cf. Farø 2004). Ein "Wörterbuch zur österreichischen Phraseologie" ist in Arbeit. Den Autoren zufolge (cf. Ernst/Peyerl 2004) basiert es auf einer Datenbank von 50.000 phraseologischen Austriazismen, von denen 10.000 bis 15.000 Phraseologismen ins Wörterbuch aufgenommen werden. Die Ermittlung phraseologischer Helvetismen stützt sich in großem Umfang auf Internetrecherchen (cf. u. a. Hofer/Schmidlin 2004; Schmidlin im Druck). Viele Phraseologismen der nationalen Varietäten haben bereits, im Unterschied zu der großen Menge der Regionalismen (cf. Abschnitt 4), in das "Variantenwörterbuch des Deutschen" (Ammon et al. 2004) Eingang gefunden.

Földes (1992) zeigt die Bandbreite der Problematik auf, die sich bei der Suche nach austriazistischen Idiomen ergeben: Manches kann außer in Österreich im gesamten oberdeutschen oder im bairisch-österreichischen Sprachraum verbreitet, anderes wiederum von Wien aus in einige (aber nicht alle) Gegenden Österreichs vorgedrungen und daher eher den Regionalismen zuzurechnen sein. Als problematisch erweist sich das plurizentrische Konzept für die Phraseologie innerhalb der (wiedervereinigten) Bundesrepublik Deutschland. Markierungen wie binnendeutsch, deutschländisch, teutonisch u.ä. werden dahingehend interpretiert, dass ein Idiom im gesamten Staatsgebiet der heutigen Bundesrepublik gültig sei, in Abgrenzung zu Austriazismen und Helvetismen. Diesem Konzept zufolge wird der Staatsgrenze die Funktion dominanter Isoglossen und dem Staatsgebiet als solchem Differenzierungscharakter zugeschrieben. Übersehen wird, dass Deutschland kein "Zentrum" aufweist, das eine ausstrahlende Kraft auf alle Regionen des Staatsraumes entwickeln konnte. Die Besonderheit der geschichtlichen Entwicklung Deutschlands liegt gerade darin, dass anstelle einer staatlichen Zentralgewalt die Macht Jahrhunderte lang von fürstlichen Landes-, d.h. Regionalgewalten ausging. Unterschiedliche kulturelle und verwaltungstechnische Strukturen sind bis heute in den Bundesländern wirksam. Auswirkungen auf die Phraseologie sind darin zu erkennen, dass einerseits viele Idiome nicht im gesamten Sprachgebiet der Bundesrepublik verbreitet und andererseits sich z. B. die relativ junge österreichisch-deutsche Staatsgrenze nicht zu einer einschneidenden Sprachgrenze entwickelt hat.

"Ideal" wäre diesem plurizentrischen Konzept zufolge eine Grenze wie die vormalige deutsch-deutsche Staatsgrenze, die nicht nur zwei Nationen, sondern zwei weltpolitische Blöcke von einander trennte und jede normale Kommunikation der Menschen beidseitig der Grenze unmöglich machte. Wie Eichhoff (1997: 199ff) anhand von großflächig vorkommenden Wörtern der Umgangssprachen aufzeigt, hatte sich jene politische Grenze 
bereits mehrfach als sprachliche Grenze erwiesen. "Hätte die Grenze noch zwei oder drei Generationen länger Bestand gehabt, so wäre es gewiß auch hier zu weitergehendem komplementärem territorialem Ausgleich gekommen" (op. cit.: 203). ${ }^{11}$ Dieses Ergebnis der Wortgeographie wird durch die areale Phraseologieforschung voll und ganz bestätigt. Im Sinne der "Plurizentrik" müsste eine vierte "(quasi)-nationale" Varietät zumindest erwähnt werden, da für den Raum der ehemaligen DDR die Herausbildung einer eigenständigen Phraseologie noch eben fassbar ist (vgl. dazu Anhang Teil I).

\section{Phraseologie in europäischen Bezügen}

Eurolinguistik ist einerseits ein aktuelles, viel versprechendes Forschungskonzept, das die europäischen Sprachen übereinzelphilologisch und unabhängig von ihrer genetischen Zugehörigkeit untersuchen möchte, andererseits ein Objekt kritisch-kontroverser Diskussionen, sofern damit ideologische, eurozentrische Vorstellungen verbunden werden. ${ }^{12}$ Obwohl auch in diesem Bereich die Phraseologie fast nie Erwähnung findet, sei auf zwei groß angelegte Forschungsprojekte kurz hingewiesen, von deren Methodenspektrum und Sichtweise Europas die Phraseologieforschung profitieren könnte: Beide Projekte gehen von der geographischen Definition Europas aus, das östlich bis zum Uralgebirge reicht und die Kaukasusregion einschließt. Die Einzelsprachen erstrecken sich auf mehrere nicht verwandte Sprachfamilien (indoeuropäisch, uralisch, altaisch, semitisch, drei autochthone KaukasusSubfamilien) sowie das isolierte Baskisch.

Zum einen ist es der 1977 begründete "Atlas Linguarum Europae" (ALE). Ziel dieses großen geolinguistischen Forschungsvorhabens ist es, die komplexe sprachliche Situation in Europa aufgrund der an 2.631 Belegstellen erhobenen Daten zu interpretieren und auf Karten zu projizieren, um in frühe kulturhistorische Schichten europäischer Lexik und die vorgeschichtliche kulturelle Vergangenheit Europas vorzudringen (cf. Viereck 2000; Viereck et al. 2003a, b). Zum anderen ist es das Projekt "Typology of Languages in Europe" (EUROTYP), das strukturelle Charakteristika, die vielen europäischen Sprachen gemeinsam sind, in großem Umfang untersucht (cf. z. B. Haspelmath et al. 2001). Zugrunde liegt die Entdeckung, dass die Sprachen Europas untereinander weitreichende Gemeinsamkeiten morphologischer, syntaktischer, semantischer Art aufweisen, die nicht auf genetische Verwandtschaft zurückzuführen sind. ${ }^{13}$ Für den vorliegenden Beitrag zur Arealphraseologie ist bemerkenswert, wie mit Hilfe der "isopleths" (cf. Dahl 2001: 1458; Haspelmath 2001: 1505), d. h. Linien, die die geographische Verbreitung gesamter Bündel sprachlicher

\footnotetext{
11 Stimmen von Forschern, die möglicherweise unter gewissen Zwängen die Existenz einer eigenständigen DDR-Varietät postulierten, sollten nicht überbewertet werden; z. B., stellvertretend für ähnliche Aussagen: "Die Veränderungen im Gebrauch der deutschen Sprache sind insgesamt so umfassend und tiefgreifend, daß sie den Fortbestand e i n e r deutschen Nationalsprache hier und in der BRD ernsthaft in Frage stellen." (Lerchner 1974: 263).

12 Vgl. z. B. Reiter (1991, 1999) sowie die Rubrik "Kontrovers diskutiert" in Leuschner (2001).

13 Für diese Phänomene vorgeschlagene Termini wie Europäischer Sprachbund und Euroversals wie auch Standard Average European (SAE) haben keine volle Akzeptanz erlangt.
} 
Merkmale andeuten, Kongruenzen vieler Sprachen auf die Karte Europas projiziert werden können.

Nur selten wird innerhalb der Typologieforschung auf Phraseologismen referiert. Vergleichbar den Sprachatlanten (vgl. Abschnitt 2) werden am ehesten Grußformeln beobachtet. Die Übereinstimmung von französisch au revoir mit deutsch auf Wiedersehen, russisch do svidanija, ungarisch a viszontlátásra usw. ist offensichtlich; sie reiche jedoch nicht aus, "um einen europäischen Sprachbund zu rechtfertigen" (König/Haspelmath 1999: 115). Eher beiläufig erwähnt Haspelmath (2001: 1506) zwei Idiome, als er die zeitliche Schichtung der strukturellen Gemeinsamkeiten den lexikalischen gegenüberstellt: "If lexical similarities between the European languages are discussed - for instance neoclassical compounding [...] or idiomatic structure (e. g. ivory tower/torre d'avorio/Elfenbeinturm, as poor as a church mouse/pauvre comme un rat d'église/arm wie eine Kirchenmaus) - then the last several centuries are the appropriate time frame for explaining the historical links, but the basic syntactic structures common to SAE languages must be older."

ALE wie auch EUROTYP haben gezeigt, dass es möglich ist, linguistische Strukturen in einer großen Anzahl von Sprachen nach den gleichen Richtlinien zu untersuchen. Im Kontrast dazu nimmt sich die Anzahl der Sprachen, die hinsichtlich ihrer Phraseologie erforscht wurden, mögen es 20 oder 25 sein, bescheiden aus. Interlinguale Gemeinsamkeiten der Phraseologie mehrerer Sprachen sind der Forschung zwar aufgefallen, jedoch eher auf zufällige Weise. Obwohl "Ähnlichkeiten" zumeist anhand einiger weniger Sprachen, z. B. im Rahmen kontrastiver Analysen, beobachtet wurden, sind hierfür ambitiöse Termini wie InterPhraseologismus oder Internationalismus zu finden. ${ }^{14}$ Diese Termini haben u. a. durch Braun/Krallmann (1990), die analoge Phraseologismen im Deutschen, Englischen, Französischen, zum Teil auch im Italienischen "entdeckt" zu haben meinten, in jüngere phraseologischen Arbeiten Verbreitung gefunden. Die Frage, weshalb gerade jene Sprachen (angesichts der etwa 6.400 Sprachen der Welt, cf. Grimes 2000) ein derart anspruchsvolles Konzept wie das des "Internationalismus" zu konstituieren vermögen, wird nicht gestellt. Vergleichbar sind Listen von Mokienko (1998) mit ähnlichen Idiomen des Englischen, Deutschen und einiger slawischer Sprachen: sie werden auch als Europäismen bezeichnet. Eine theoretische Fundierung oder Arbeitsdefinition dieses Terminus, ein Kriterienkatalog zur Erfassung "phraseologischer Europäismen" sowie systematische Untersuchungen zur Verbreitung von Idiomen, unter Einbeziehung möglichst vieler Sprachen, existierten bis vor kurzem nicht. Im Anhang Teil II wird ein Projekt vorgestellt, das zum Ziel hat, ein Inventar der in vielen Sprachen verbreiteten Idiome zu erstellen. Erste Ergebnisse zeigen bereits die Existenz weit verbreiteter Idiome, sogar über Europa hinaus: So wurde das Idiom Krokodilstränen weinen/vergießen, das auf sehr alte, weitgehend in Vergessenheit geratene Fabeltraditionen zurückzuführen ist, nicht nur in fast alle europäischen Sprachen (abgesehen

14 Internationalismus findet sich zuvor vereinzelt in phraseologischen Studien, ebenfalls ohne Begriffsbestimmung. So nennt Ehegötz (1973: 229) für den deutsch-polnischen Sprachvergleich "die große Zahl der 'phraseologischen Internationalismen': dolać oliwy do ognia - 'Öl ins Feuer gießen'". Rajxštejn (1980: 36, 125) spricht von ФЕ-интернаиионализмы für Idiome wie den Augiasstall ausfegen, das Trojanische Pferd, das goldene Kalb anbeten. 
von einigen Kleinsprachen), sondern auch in Sprachen Asiens und Afrikas verbreitet (cf. Piirainen 2005: 62ff).

\section{Ausblick}

Mit diesem Beitrag sollen die arealen Faktoren der Phraseologie stärker ins Blickfeld gerückt und die Gleichsetzung von "Sprache" und "Raum" der klassischen Phraseologieforschung problematisiert werden, sowohl nach innen, da Idiome (oft aufgrund ihrer dialektalen Herkunft) regional begrenzt gültig sein können (cf. dazu Anhang Teil I), als auch nach außen, da bestimmte Idiome in weit größeren Arealen als dem einer Einzelsprache existieren (vgl. dazu Anhang Teil II). Die Beobachtungen zu den Verbreitungstypen von Idiomen (innerhalb der bodenständigen Dialekte, der kleinräumig-regionalen Umgangssprachen, der nationalen Varianten und eines sprachübergreifenden Raumes Europa und darüber hinaus) stehen in Beziehung zu einander. Der Transfer (d.h. die wortwörtliche Übernahme) basisdialektaler Idiome in die örtlichen Umgangssprachen, wie er vielerorts in Deutschland zu verfolgen ist, könnte als ein Modell auch zur Erklärung anderer Verbreitungstypen herangezogen werden. Vergleichbare kontaktbedingte Übersetzungs-Transferenzen aus überdachenden oder angrenzenden großen Standardsprachen in die kleineren, weniger vitalen Sprachen sind europaweit zu erkennen (z. B. Englisch $\rightarrow$ Kymrisch, Französisch $\rightarrow$ Bretonisch, Polnisch $\rightarrow$ Kaschubisch usw.).

Für die weite Verbreitung (vor allem bildungssprachlicher) Idiome muss hinzugefügt werden, dass sie weniger auf mündliche Sprachkontakte als vor allem auf den gemeinsamen Bildungskodex der lesekundigen europäischen Bildungsschicht zurückzuführen sind. Intensiver Gedankenaustausch über viele unterschiedliche Sprachen hinweg konnte nur auf dem Wege des Lesens und Schreibens vonstatten gehen, vorwiegend mittels des Lateinischen als Universalsprache. Der Vergleich der unterschiedlich weit verbreiteten Idiome ergibt ein Weiteres: In vielen Fällen geht es nicht darum, Idiome eines ortsgebundenen, nur mündlich existierenden Dialektes einer wenig mobilen Sprechergemeinschaft mit den Idiomen der/einer Standardsprache zu vergleichen, sondern darum, die Besonderheiten der dialektalen Phraseologie im Kontrast zur relativ uniformierten Phraseologie (europäischer) Standardsprachen mit einer langen Schrifttradition herauszuarbeiten.

Die Konsequenzen für die Phraseologieforschung sind offensichtlich: Ein Bewusstsein für die Arealität von Idiomen sollte sowohl in die Theoriebildung einbezogen als auch in die Praxis (Wörterbücher, Fremdsprachen-Lehrwerke) umgesetzt werden. Die hier aufgezeigten Fragestellungen können nur auf der Basis umfassender empirischer Untersuchungen erörtert werden. Ein Ziel der Bemühungen wäre es - in Analogie zu der eingangs erwähnten griechischen Anekdote -, ein Bild von der Sprachwirklichkeit nicht durch Intuition, sondern durch Anschauung der empirisch bereitgestellten Daten zu gewinnen, wofür allerdings noch viele Vorarbeiten zu leisten sind. 


\section{Literaturangaben}

Abraham, Werner/Bayer, Josef (1993): "Einleitung". In: Abraham, Werner/Bayer, Josef (eds.): Dialektsyntax. Opladen: 7-11.

Ammon, Ulrich et al. (2004): Variantenwörterbuch des Deutschen. Die Standardsprache in Österreich, der Schweiz und Deutschland sowie in Liechtenstein, Luxemburg, Ostbelgien und Südtirol. Berlin/New York.

Braun, Peter/Krallmann, Dieter (1990): "Inter-Phraseologismen in europäischen Sprachen". In: Braun, Peter et al. (eds.): Internationalismen. Studien zur interlingualen Lexikologie und Lexikographie. Tübingen: 74-86.

Burger, Harald (1995): "Helvetismen in der Phraseologie". In: Löffler, Heinrich (ed.): Alemannische Dialektforschung - Bilanz und Perspektiven. Beiträge zur 11. Arbeitstagung alemannischer Dialektologen. Tübingen/Basel: 13-25.

Burger, Harald (1996): "Zur Phraseologie des Schweizerhochdeutschen". In: Korhonen, Jarmo (ed.): Studien zur Phraseologie des Deutschen und des Finnischen II. Bochum: 461-488.

Burger, Harald (1998): "Helvetismen in der Phraseologie - Vorkommen und stilistische Funktionen". In: Hartmann, Dietrich (ed.): "Das geht auf keine Kuhhaut". Arbeitsfelder der Phraseologie. Akten des Westfälischen Arbeitskreises Phraseologie/Parömiologie 1996. Bochum: 49-80.

Burger, Harald (2002): Dialektale Phraseologie - am Beispiel des Schweizerdeutschen. In: Piirainen, Elisabeth/Piirainen, Ilpo Tapani (eds.): Phraseologie in Raum und Zeit. Akten der 10. Tagung des Westfälischen Arbeitskreises "Phraseologie/Parömiologie" Münster 2001. Baltmannsweiler: 11-29.

Burger, Harald (2003): Phraseologie. Eine Einführung am Beispiel des Deutschen. Berlin.

Burger, Harald et al. (eds.) (im Druck): Phraseology. An International Handbook of Contemporary Research. Berlin/New York.

Chambers, Jack K./Trudgill, Peter ( $\left.{ }^{2} 2002\right)$ : Dialectology. Cambridge.

Clyne, Michael (1984): Language and Society in the German Speaking Countries. Cambridge.

Crede, Claudia/Lakemper, Udo (1998): "Empirische Untersuchungen zur Phraseologie im Ruhrgebiet". In: Hartmann, Dietrich (ed.) "Das geht auf keine Kuhhaut". Arbeitsfelder der Phraseologie. Akten des Westfälischen Arbeitskreises Phraseologie/Parömiologie 1996. Bochum: 81-108.

Dahl, Östen (2001): "Principles of areal typology". In: Haspelmath, Martin et al. (eds.): Language Typology and Language Universals. An International Handbook. Vol. 2. Berlin/New York: 1456-1470.

Dawes, Elizabeth (2003): "Étude contrastive de la phraséologie française et québécoise". In: Burger, Harald et al. (eds.): Flut von Texten - Vielfalt der Kulturen. Ascona 2001 zur Methodologie und Kulturspezifik der Phraseologie. Baltmannsweiler: 183-191.

Dobrovol'skij, Dmitrij O./Piirainen, Elisabeth (2005): Figurative Language: Cross-cultural and Cross-linguistic Perspectives. Amsterdam etc. 
Duden 11 (1998): Duden. Redewendungen und sprichwörtliche Redensarten. Wörterbuch der deutschen Idiomatik. Bearb. von Günther Drosdowski und Werner Scholze-Stubenrecht. Mannheim etc.

Duden 11 (2002): Duden. Redewendungen. Wörterbuch der deutschen Idiomatik. 2., neu bearb. Auflage. Herausgegeben von der Dudenredaktion. Mannheim etc.

Ebata, Yoshio (2003): A Completion of Courtesy Atlas in Japanese Greetings and International Exchange. Research Papers of the Pedagogical Faculty, Project no. 11610435. Hiroshima. [auf Japanisch].

Eggers, Eckhard et al. (eds.) (2005): Moderne Dialektologie - Neue Dialektologie. Akten des 1. Kongresses der Internationalen Gesellschaft für Dialektologie des Deutschen (IGDD). Stuttgart.

Ehegötz, Erika (1973): "Zur Konzeption eines polnisch-deutschen phraseologischen Wörterbuchs". Zeitschrift für Slawistik 18: 227-234.

Eichhoff, Jürgen (1977ff): Wortatlas der deutschen Umgangssprachen. Bd. 1 (1977), Bd. 2 (1978) Bern/München, Bd. 3 (1993), Bd. 4 (2000) München etc.

Eichhoff, Jürgen (1997): "Der 'Wortatlas der deutschen Umgangssprachen': Neue Wege, neue Erkenntnisse". In: Stickel, Gerhard (ed.): Varietäten des Deutschen: Regional- und Umgangssprachen; Jahrbuch des Instituts für Deutsche Sprache 1996. Berlin/New York: 183-220.

Eismann, Wolfgang (1991): "Zur Frage der lexikographischen Berücksichtigung von nichtbinnendeutschen Phraseologismen in deutsch-slavischen phraseologischen Wörterbüchern". In: Palm, Christine (ed.): "Europhras 90". Akten der internationalen Tagung zur germanistischen Phraseologieforschung Aske. Schweden 12.-15. Juni 1990. Uppsala: 43-61.

Elspaß, Stephan/Möller, Robert (2003ff): Atlas zur deutschen Alltagssprache (AdA). http://www.philhist.uni-augburg.de/ada.

Ernst, Peter/Peyerl, Elke (2004): "Das 'Wörterbuch zur österreichischen Phraseologie'. Eine Projektbeschreibung". In: Földes, Csaba (ed.): Res humanae proverbiorum et sententiarum. Ad honorem Wolfgangi Mieder. Tübingen: 79-88.

Farø, Ken (2004): "Verbildete, verbeamtete und überalterte Deutschschweizer? Das Schweizerhochdeutsche im Wörterbuch und sonst". In: Williams, Geoffrey/Vessier, Sandra (eds.): Proceedings of the Eleventh EURALEX International Congress. Bretagne: 879-884.

Filatkina, Natalia (2005): Phraseologie des Lëtzebuergeschen. Empirische Untersuchungen zu strukturellen, semantisch-pragmatischen und bildlichen Aspekten. Heidelberg.

Filatkina, Natalia (in Vorbereitung): "Moselfränkisch diesseits und jenseits der Staatsgrenze". Filppula, Markku et al. (eds.) (2005): Dialects Across Borders: Selected Papers from the 11th International Conference on Methods in Dialectology (Methods XI), Joensuu, August 2002. Amsterdam etc.

Fleischer, Wolfgang (1982): Phraseologie der deutschen Gegenwartssprache. Leipzig.

Fleischer, Wolfgang (ed.) (1987): Wortschatz der deutschen Sprache in der DDR. Fragen seines Aufbaus und seiner Verwendungsweise. Leipzig. 
Földes, Csaba (1992): "Zu den österreichischen Besonderheiten der deutschen Phraseologie". In: Földes, Csaba (ed.): Deutsche Phraseologie in Sprachsystem und Sprachverwendung. Wien: 9-24.

Földes, Csaba (1996): Deutsche Phraseologie kontrastiv. Intra- und interlinguale Zugänge. Heidelberg.

Goosens, Jan (1980): "Areallinguistik". In: Althaus, Peter et al. (eds.): Lexikon der Germanistischen Linguistik. Tübingen: 445-453.

Grimes, Barbara (ed.) (2000): Ethnologue. Languages of the World. Dallas.

Grober-Glück, Gerda (1974): Motive und Motivationen in Redensarten und Meinungen. Aberglaube, Volks-Charakterologie, Umgangsformeln, Berufsspott in Verbreitung und Lebensformen. Marburg.

Haspelmath, Martin (2001): "The European linguistic area: Standard Average European". In: Haspelmath, Martin et al. (eds.): Language Typology and Language Universals. An International Handbook. Vol. 2. Berlin/New York: 1492-1510.

Haspelmath, Martin et al. (eds.) (2001): Language Typology and Language Universals. An International Handbook. Vol. 2. Berlin/New York.

Hofer, Lorenz/Schmidlin, Regula (2004): "Variants of phraseological units in a dictionary of national varieties of Standard German". In: Allerton, David J. et al. (eds.): Phraseological Units: basic concepts and their application. Basel: 171-184.

Knop, Kerstin (in Vorbereitung) "Der kennt e Gääß zwische de Hörner kisse - Zu einigen Besonderheiten der Phraseologie des Pfälzischen". (Beitrag zur Europhras-Tagung in Veszprém, 9.-11. Juni 2006).

König, Ekkehard/Haspelmath, Martin (1999): "Der europäische Sprachbund". In: Reiter, Norbert (ed.): Eurolinguistik: Ein Schritt in die Zukunft. Beiträge zum Symposion vom 24. bis 27. März 1997 im Jagdschloß Glienicke (bei Berlin). Wiesbaden: 111-127.

Kretschmer, Paul (1918): Wortgeographie der hochdeutschen Umgangssprache. Göttingen.

Lerchner, Gotthard (1974): "Zur Spezifik der Gebrauchsweise der deutschen Sprache in der DDR und ihre gesellschaftliche Determinination". Deutsch als Fremdsprache 11/5: 259-254.

Leuschner, Torsten (ed.) (2001): "Sprachkontaktforschung und Areallinguistik: Europa. Language contact and areal linguistics: Europe". Linguistik online 8, 1/01.

Löffler, Heinrich (2003): Dialektologie. Eine Einführung. Tübingen.

Makkai, Adam (1972): Idiom structure in English. The Hague/Paris.

Matešič, Josip/Petermann, Jürgen (1987): "Zur Problematik der arealen Phraseologie am Beispiel des Kroatischen, Russischen und Deutschen". In: Burger, Harald/Zett, Robert (eds.): Aktuelle Probleme der Phraseologie. Symposium 27.-29. 9. 1984 in Zürich. Bern etc.: 259-265.

Meney, Lionel (1999): Dictionnaire québécois français. Montréal.

Mitzka, Walther et al. (1951-1980): Deutscher Wortatlas. 22 Bde. Giessen.

Mokienko, Valerij M. (1998): "Phraseologisierung von Europäismen oder Europäisierung von Phraseologismen? Divergente und konvergente Prozesse in phraseologischen Systemen europäischer Sprachen". In: Eismann, Wolfgang (ed.): Europhas 95. Europäische Phraseologie im Vergleich: Gemeinsames Erbe und kulturelle Vielfalt. Bochum: 539-555. 
Niebaum, Hermann/Macha, Jürgen (2006): Einführung in die Dialektologie des Deutschen. Tübingen. 2., neu bearbeitete Auflage. Tübingen.

Palm, Christine (1995): Phraseologie. Eine Einführung. Tübingen.

Piirainen, Elisabeth (1999): "Dat is doch mäer, äs int Näppken geiht! Idiome im Frühwerk Augustin Wibbelts". Jahrbuch der Augustin Wibbelt-Gesellschaft 15: 21-37.

Piirainen, Elisabeth (2000): Phraseologie der westmünsterländischen Mundart. Teil 1: Semantische, kulturelle und pragmatische Aspekte dialektaler Phraseologismen. Teil 2: Lexikon der westmünsterländischen Redensarten. Baltmannsweiler.

Piirainen, Elisabeth (2002a) "'Landschaftlich', 'norddeutsch' oder 'berlinisch'? Zur Problematik diatopischer Markierungen von Idiomen". Deutsch als Fremdsprache 39/1: 36-40.

Piirainen, Elisabeth (2002b) "Er zahlt keine Steuern mehr. Phraseologismen für 'sterben' in den deutschen Umgangssprachen". In: Piirainen, Elisabeth/Piirainen, Ilpo Tapani (eds.): Phraseologie in Raum und Zeit. Akten der 10. Tagung des Westfälischen Arbeitskreises "Phraseologie/Parömiologie" Münster 2001. Baltmannsweiler: 213-238.

Piirainen, Elisabeth (2002c) "Ein Wink mit dem Scheunentor? Nochmals zur Bekanntheit von Idiomen". Deutsch als Fremdsprache 39/4: 221-225.

Piirainen, Elisabeth (2002d) "Bier mit ins Hofbräuhaus nehmen oder Sand in die Sahara tragen? Nochmals zum Idiomtyp 'Eulen nach Athen tragen'". Proverbium. Yearbook of International Proverb Scholarship 19: 349-359.

Piirainen, Elisabeth (2003a): "Es ist noch nicht im Topf, wo's kocht. Zu Idiomen aus dem Raum der ehemaligen DDR". Niederdeutsches Wort. Beiträge zur niederdeutschen Philologie 43: 203-219.

Piirainen, Elisabeth (2003b): "Areale Aspekte der Phraseologie: Zur Bekanntheit von Idiomen in den regionalen Umgangssprachen". In: Burger, Harald et al. (eds.): Flut von Texten Vielfalt der Kulturen. Ascona 2001 zur Methodologie und Kulturspezifik der Phraseologie. Baltmannsweiler: 117-128.

Piirainen, Elisabeth (2004a): "Cognitive, Cultural and Pragmatic Aspects of Dialectal Phraseology - Exemplified by the Low German Dialect 'Westmünsterländisch'". Dialectologia et Geolinguistica 12: 78-99.

Piirainen, Elisabeth (2004b): "Er poliert seinen Heiligenschein. Zu Idiomen des semantischen Feldes 'Frömmigkeit'". In: Kiedroń, Stefan/Kowalska-Szubert, Agata (eds.): Thesaurus polyglottus et flores quadrilingues. Festschrift für Stanisław Prędota zum 60. Geburtstag. Wrocław: 409-421.

Piirainen, Elisabeth (2005): "Europeanism, internationalism or something else? Proposal for a cross-linguistic and cross-cultural research project on widespread idioms in Europe and beyond". Hermes. Journal of Linguistics 35: 45-75.

Piirainen, Elisabeth (2006): "Widespread Idioms: Cross-linguistic and Cross-cultural Approaches". In: Häcki Buhofer, Annelies/Burger, Harald (eds.): Phraseology in Motion I. Methoden und Kritik. Akten der Internationalen Tagung zur Phraseologie (Basel 2004). Baltmannsweiler: 155-173.

Piirainen, Elisabeth (im Druck a): "Dialectal phraseology - linguistic aspects". In: Burger, Harald et al. (eds.) Phraseology. An International Handbook of Contemporary Research. Berlin/New York. 
Piirainen, Elisabeth (im Druck b): "Phraseologie in europäischen Bezügen: zu einem Forschungsprojekt". In: Kržišnik, Erika (ed.): Phraseologie in der Sprachwissenschaft und anderen Disziplinen (Akten der Europhras-Tagung in Strunjan/Slowenien, 19.-22. September 2005).

Rajxštejn, Aleksandr D. (1980): Sopostavitel'nyj analiz nemeckoj i russkoj frazeologii. Moskva.

Reiter, Norbert (1991): "Ist Eurolinguistik Gotteslästerung?" In: Feldbusch, Elisabeth et al. (eds.): Neue Fragen der Linguistik. Akten des 25. Linguistischen Kolloquiums, Paderborn 1990. Band 1: Bestand und Entwicklung. Tübingen: 109-113.

Reiter, Norbert (ed.) (1999): Eurolinguistik: Ein Schritt in die Zukunft. Beiträge zum Symposion vom 24. bis 27. März 1997 im Jagdschloß Glienicke (bei Berlin). Wiesbaden.

Schmidlin, Regula (2004): "Nationale Varianten standarddeutscher Phraseologismen". In: Palm-Meister, Christine (ed.): Europhras 2000. Internationale Tagung zur Phraseologie vom 15.-18. Juni 200 in Aske/Schweden. Tübingen: 435-446.

Schmidlin, Regula (im Druck): "Phraseme in standardsprachlichen Varietäten des Deutschen. Phraseological expressions in German standard varieties". In: Burger, Harald et al. (eds.): Phraseology. An International Handbook of Contemporary Research. Berlin/New York.

Schönfeld, Helmut/Schlobinski, Peter (1995): "After the Wall: Social Change and Linguistic Variation in Berlin". In: Stevenson, Patrick (ed.): The German Language and the Real World. Sociolingustic, Cultural, and Pragmatic Perspectives on Contemporary German. Oxford: 117-134.

Sternemann, Reinhard/Gutschmidt, Karl (1989): Einführung in die vergleichende Sprachwissenschaft. Berlin.

Viereck, Wolfgang (2000): "The Atlas Linguarum Europae and its Insights into the Cultural History of Europe". In: Gottlieb, Henrik et al. (eds.): Symposium on Lexicography X. Proceedings of the Tenth International Symposium on Lexicography May 4-6, 2000 at the University of Copenhagen. Tübingen: 19-29.

Viereck, Wolfgang et al. (eds.) (2003a): Atlas Linguarum Europae: Vol. I, sixième fascicule. Cartes. Published under the auspices of UNESCO. Roma.

Viereck, Wolfgang et al. (eds.) (2003b): Atlas Linguarum Europae: Vol. I, sixième fascicule. Commentaires. Published under the auspices of UNESCO. Roma.

Wenker, Georg (1881): Sprach-Atlas von Nord- und Mitteldeutschland: Text und Einleitung: auf Grund von systematisch mit Hülfe der Volksschullehrer gesammeltem Material aus circa 30000 Orten. Bearb., entworfen u. gezeichnet von G. Wenker. Strassburg.

Wolf, Birgit (2000): Sprache in der DDR: ein Wörterbuch. Berlin/New York.

Zürrer, Peter (im Druck): "Phraseme aus germanistisch-dialektologischer Sicht". In: Burger, Harald et al. (eds.): Phraseology. An International Handbook of Contemporary Research. Berlin/New York.

Zürrer, Peter/Burger, Harald (2004): "Phraséologie en région alpine - Phraseology in the Alpine area". In: Baccouche, Taieb et al. (eds.): L'espace euro-méditerranéen: une idiomaticité partagée. Hammamet, 19-21 septembre. Rencontres linguistiques méditerranéennes/Europhras. Tunis: 463-474. 


\section{Anhang}

\section{Teil I: Projekt "Umfrage zur Bekanntheit von Redensarten"}

\section{$1 \quad$ Anlage und Auswertung}

In den Jahren 2000 und 2001 wurden Erhebungen zur Bekanntheit von Idiomen unter dem Aspekt der Diatopik im Raum der Bundesrepublik Deutschland durchgeführt. ${ }^{15}$ Den Gegenstand bildeten Idiome, für die eine areale Begrenzung vermutet wurde (die als "fraglich" aufgefallen waren, cf. Abschnitt 4). In zwei Etappen wurden Fragebögen versandt, vorwiegend noch auf postalischem Wege, und zwar an zwei Zielgruppen, die jeweils als Multiplikatoren tätig wurden: an rund 300 Dozierende an den 90 Germanistischen Instituten der Universitäten und an die rund 30 Landeskundlichen Institute in Deutschland. In einem Anschreiben wurde darum gebeten, Fotokopien der Fragebögen möglichst vielen Personen aus allen Regionen Deutschlands zur Begutachtung vorzulegen. Es ergab sich ein Rücklauf von jeweils rund 3.000 Fragebögen aus allen Gegenden Deutschlands, so dass die Umfrage für die Zielsetzungen im statistischen Sinne repräsentativ ist.

Teil A des ersten 10-seitigen Fragebogens umfasst 75 Idiome. Es standen die Rubriken 1. "bekannt", 2. "unbekannt" sowie 3. "schon mal gehört, aber ich gebrauche den Ausdruck selbst nicht" zur Verfügung. Diese dritte Rubrik bot die Möglichkeit, sich nicht unbedingt zu entscheiden, um den Ja-/Nein-Effekt nicht zu verfälschen, sowie zugleich Raum für zusätzliche Eintragungen. Sie erwies sich als überaus ergiebig, da dort viele Varianten, weitere Bedeutungsangaben und bisher nicht lexikographisch erfasste Idiome mitgeteilt wurden. In Teil B wurden 96 Idiome angeführt, die sich Voruntersuchungen zufolge als Heteronyme erweisen könnten (z. B. seinen Dreier dazugeben vs. seinen Senf dazugeben), wobei mehrere Ja-Antworten möglich waren. Auch hier wurden viele zusätzliche Ausdrücke eingetragen. Etwa ein Jahr später wurde der ähnlich strukturierte zweite Fragebogen verschickt, der gezielt neu aufgetauchte Problemfälle aufgreift. ${ }^{16}$

Im Vorspann der Fragebögen wurde nach den derzeitigen und früheren Wohnorten der Probanden/innen gefragt. Es ergaben sich die drei Gruppen der stets am gleichen Ort wohnhaften Personen, der wenig mobilen Personen und der sehr mobilen Personen, jeweils unterteilt in die Altersgruppen bis 29 Jahre und über 29 Jahre. Rund 1900 Fragebögen, also fast zwei Drittel, wurden von Studierenden im Alter von 19 bis 29 Jahren ausgefüllt. Sie waren zumeist noch in ihrer heimatlichen Gegend wohnhaft und wenig mobil, gehörten also den Herkunftsgruppen 1 und 2 an. Diese in allen Regionen Deutschlands alters- und bildungshomogene Gruppe war für die Auswertung hinsichtlich des arealen Aspekts der

\footnotetext{
${ }^{15}$ In Zusammenarbeit mit dem Institut für deutsche Sprache und Literatur und ihre Didaktik der Universität Münster. Die Beschränkung auf die Bundesrepublik hatte organisatorische Gründe.

16 Beide Fragebögen enthalten zusätzlich einen Teil C, in dem nach Redewendungen bestimmter Wortfelder gefragt wird ('Sterben', 'Armut', 'Frömmigkeit'). Es ergab sich eine Fülle zuvor nicht erfasster Ausdrücke; cf. dazu u. a. Piirainen (2002b, c, d, 2003b, 2004b).
} 
Idiom-Kenntnisse besonders hilfreich, da nur der Faktor "Herkunft/Wohnort" nicht einheitlich war.

Eines der Ziele der Untersuchung war es, die Daten der Fragebögen der geographischen Karte Deutschlands zuzuordnen. Deutschland wurde in 27 Planflächen, diese wiederum in vier kleinere Flächen (a, b, c, d) unterteilt. So ergibt sich für Husum der Ortscode "1-a", für Freilassing der Code "27-d". Die Wohnorte der Probanden/innen der Herkunftsgruppen 1 und 2 wurden auf der Deutschlandkarte gesucht, die Fragebögen sodann mit dem Ortscode versehen, dem eine Einheit der Karte entspricht. Hierin lag der wichtigste Arbeitsschritt, um die Idiom-Bekanntheit auf den Raum zu projizieren. Die sich anschließenden Arbeitsschritte wie die quantitativen und prozentualen Berechnungen zur Bekanntheit des Idioms pro Planfläche konnten weitgehend computerintern vorgenommen werden. Eine relationale Datenbank ermöglicht die Auswertungen nach verschiedenen Gesichtspunkten (ausführlicher dazu Piirainen 2003a, b).

\section{Ergebnisse für die germanistische Phraseologieforschung}

Zwei der zahlreichen Ergebnisse dieser Umfragen seien hier hervorgehoben: 1. die Existenz arealphraseologischer Heteronymie ${ }^{17}$ und 2. die noch eben fassbare Existenz einer eigenen phraseologischen DDR-Varietät: beides war in der Forschung zuvor in dieser Deutlichkeit nicht bekannt.

Beide Ergebnisse lassen sich anhand der Kartierung des Idioms etwas für lau tun/haben wollen 'etwas umsonst tun/haben wollen' veranschaulichen (Karte 1). Das Kartenbild zeigt, dass für lau - in der Reihenfolge der Planflächen - erstmals im Süden der Fläche 8-c eine relevante Belegdichte erreicht, gefolgt von 8-d und den meisten Flächeneinheiten von 9, 12, 13, 17, 18 usw. Die Karte zeigt einen arealen Schwerpunkt in der mittleren westlichen Bundesrepublik, vom Ruhrgebiet bis nordöstlich von Hannover und südwestlich bis ins Saarland. Der Umfrage zufolge handelt es sich also um einen Regionalismus und nicht, wie mehrfach behauptet wurde (z. B. Schmidlin 2004: 435), um einen Teutonismus.

Die Fragebögen zeigten ein Weiteres. In der dritten Rubrik wurden häufig zwei quasisynonyme Idiome angeführt, und zwar etwas für umme tun/haben wollen für den RheinNeckar-Raum sowie etwas für nass(e) tun/haben wollen für den Raum Obersachsen und Thüringen. Das Idiom für umme ist in den Wörterbüchern bisher nicht verzeichnet, in der Umgangssprache jener Region jedoch recht lebendig..$^{18}$ Dagegen findet sich für nass(e) in vielen Wörterbüchern Leipziger oder Ostberliner Provenienz (vgl. Anmerkung 8). Das Variantenwörterbuch (Ammon et al. 2004) kennt jedoch weder für umme noch für nass(e).

17 Der in der Wortgeographie übliche Terminus Heteronymie lässt sich somit auch auf die Phraseologieforschung anwenden, die noch keine Terminologie für areale Phänomene entwickelt hat.

18 In den COSMAS-Korpora des IDS Mannheim ist für umme mit ca. 80 Beispielen aus dem "Mannheimer Morgen" vertreten, da Mannheim im Einzugsbereich dieses Idioms liegt. Andere lokale Zeitungen wurden nicht in gleicher Weise in die Korpora aufgenommen, so dass sich für für nass(e) und andere, seltener gemeldete Entsprechungen (etwas für noppes/für nüsse haben/tun wollen) dort keine Belege finden. 
Die Projektion der Bekanntheit dieser beiden Idiome auf die Karte beruht auf der zweiten Umfrage. Karte 1 zeigt nicht nur die drei klar umrissenen phraseologischen Areale, sondern vor allem, wie für lau und für nass(e) jeweils vor der einstigen deutsch-deutschen Grenze Halt machen. Darüber hinaus ist für das vormals nur in der südlichen DDR geläufige für nass(e) die Ausstrahlung nach Mecklenburg und Berlin (Ost) zu erkennen.

Dieses Beispiel ist kein Einzelfall. Die 41 Jahre währende Teilung Deutschlands mag sprachhistorisch als eine kurze Phase erscheinen, doch kennt die Geschichte kaum Vergleichbares. Die Intensität dieser Teilung hat deutliche Spuren in der Phraseologie hinterlassen. Es findet sich ein Bündel von (vormals) eigenständigen, nur in der DDR bekannten Idiomen, die bei der Kartierung ein ähnliches Bild wie für nass $(e)$ ergeben. Viele sind zunächst, auf ein dialektales Substrat zurückzuführende, Regionalismen des Raumes Thüringen-Obersachsen, die in den Norden der DDR expandierten, jedoch nicht über die deutsch-deutsche Grenze nach Westen gelangten. ${ }^{19}$ So ist - aufgrund der völligen Abgeschlossenheit der einstigen DDR - diese vierte Varietät mit einem "Zentrum" (dem industriellen Ballungsraum Obersachsen) noch eben greifbar, was in anderen Teilen Deutschlands, z. B. dem vergleichbaren Ballungszentrum Ruhrgebiet, nicht mehr so eindeutig möglich ist.

Weitere Ergebnisse gruppieren sich um die große Variabilität der Idiome und die Fülle der zusätzlich mitgeteilten in den Umgangsprachen existierenden Ausdrücke, von denen nur ein Bruchteil in den Wörterbüchern erfasst wurde. Allein das Beispiel erzähl mir doch keinen vom Pferd erbrachte rund 50 Varianten oder zusätzliche Benennungen. ${ }^{20}$

\section{Anhang}

\section{Teil II: Projekt "Weit verbreitete Idiome in Europa und darüber hinaus"}

\section{$1 \quad$ Vorgehensweisen und Ziele}

Erste Überlegungen zu einem Forschungsvorhaben, als "weit verbreitet" vermutete Idiome systematisch zu erfassen, führen auf die Phraseologietagung in Basel im August 2004 zurück. Zugrunde lag der Gedanke, dass es in der heutigen Zeit, im sich vereinigenden Europa, möglich sein müsste, für sehr viele Sprachen - zumindest für die in den demokratisch regierten Ländern Europas gesprochenen Sprachen - kompetente Mitarbeiter/innen zu gewinnen. Das Projekt "Widespread Idioms in Europe and Beyond", das von der

\footnotetext{
19 In den seit 1990 erschienenen Monographien zur Sprache der DDR werden Phraseologismen nur selten berücksichtigt. Schönfeld/Schlobinski (1995: 131) nennen sich keinen Kopf machen und das ist Fakt; in Wolf (2000) werden einige Idiome erwähnt, die auf Realien jener DDR-Zeit referieren, z. B. dumm wie Konsumbrot oder (Zettel) falten gehen (zur Wahl gehen). Aufgrund der Ergebnisse der Umfragen werden 32 weitere DDRspezifische Idiome analysiert (Piirainen 2003a).

${ }^{20}$ Z. B. erzähl mir doch keinen vom rosa Pferd/nichts vom Pferd/nicht die Geschichte vom Pferd/nicht die Story vom Pferd/die Story vom blauen Pferd/ die Geschichte vom toten Pferd usw.; erzähl mir doch keinen Wolf/keinen Gruß/kein Kind in den Bauch/nicht was vom Storch/nix vom Fisch/erzähl mir keinen vom Horst/nicht die Geschichte vom Krieg/das Märchen vom Krieg/schwätz mir doch keine Vesper in den Rucksack u. a. m.
} 
Europäischen Gesellschaft für Phraseologie unterstützt wird, setzt sich zum Ziel, ein Inventar der tatsächlich existierenden in vielen Sprachen verbreiteten Idiome zu erstellen, um Fragen zum Anteil der Phraseologie an der beobachteten Uniformität der Sprachen Europas nicht mehr aufgrund von Zufälligkeiten, sondern auf einer empirisch gesicherten Basis beantworten zu können. Statt Europäismus oder Internationalismus wird der neutrale Terminus weit verbreitetes Idiom, englisch widespread idiom (kurz: WI) gewählt, der frei ist von eurozentrischen Assoziationen. Tatsächlich können WIs oft sowohl in Sprachen Europas als auch in Sprachen anderer Kontinente nachgewiesen werden. ${ }^{21}$

Unter weit verbreiteten Idiomen/widespread idioms werden in mehreren Sprachen, darunter in genetisch nicht verwandten und geographisch nicht benachbarten, in der gleichen bzw. einer sehr ähnlichen lexikalischen Struktur und in der gleichen figurativen Bedeutung vorkommende Idiome verstanden (Arbeitsdefinition). Der erste Schritt zur Ermittlung von WIs besteht in den Vortests: Idiome, die als weit verbreitet gelten könnten, werden zunächst anhand mehrerer genetisch und geographisch unterschiedlicher Sprachen überprüft; außer Englisch, Französisch, Deutsch sind dies zunächst Finnisch, Ungarisch und Griechisch, gefolgt von Russisch, Bulgarisch, Rumänisch und Spanisch. Viele als WI vermutete Idiome fallen durch das Raster dieser Vortests.

Für die auf diese Weise ermittelten "WI-Kandidaten" werden Fragebögen entworfen, diese sodann per E-Mail an Experten/innen möglichst vieler Sprachen verschickt mit der Bitte, die Fragen aufgrund der eigenen Idiom-Kompetenz sowie im Kreise von Kollegen/innen zu begutachten. Jeder Zielgruppe entsprechend werden die Fragebögen und Anschreiben in verschiedenen Sprachen und Versionen individuell gestaltet. In zwei Etappen wurden Fragebögen mit insgesamt 100 vermutlich weit verbreiteten Idiomen an Mitarbeiter/innen für rund 80 Sprachen verschickt. Bis jetzt haben sich über 120 Personen an dem Projekt beteiligt. Eine zuverlässige Unterstützung fand das Projekt ferner seitens landeskundlicher Forschungsinstitute speziell einiger europäischen Kleinsprachen.

Für 70 Sprachen liegen zuverlässige Daten vor: Indoeuropäisch ist mit 53 (der für die Forschung zugänglichen) Sprachen fast vollständig vertreten, gleiches gilt für Maltesisch und Baskisch. Schwieriger ist die Datenerhebung der uralischen Sprachen. Der ugrische Sprachzweig ist nur durch Ungarisch, der nordseefinnische nur durch Finnisch, Karelisch und Estnisch, der permischen Zweig nur durch Udmurtisch vertreten. Es wird weiterhin versucht, an Probanden/innen der noch fehlenden finnisch-ugrischen Sprachen zu gelangen. Problematisch sind auch die altaischen Sprachen. Zuverlässige Daten für Kalmykisch und die z. T. moribunden südwestlichen Turksprachen Europas zu bekommen, wird nicht möglich sein, doch wird Tatarisch von Mitgliedern der Gesellschaft Europhras bearbeitet. Von den ca. 60 autochthonen Kaukasussprachen ist nur Georgisch im Projekt vertreten. Als außereuropäische Kontrastsprachen wurden bisher Persisch, Telugu, Hindi, Chantisch, Koreanisch, Japanisch, Chinesisch und Vietnamesisch herangezogen. Auch für Esperanto und verschiedene Dialekte wurden die Fragebögen ausgefüllt.

\footnotetext{
${ }^{21}$ Ausführliche Beispiele dazu finden sich in Piirainen (2005, 2006, im Druck b).
} 


\section{Präsentation der Daten}

Da das WI-Projekt in der Areallinguistik angesiedelt ist, verlangt es nach einer Projektion der Daten auf die Karte Europas. Angesichts mehrerer sich räumlich überlappender Sprachen können nur schematische Vorkommenskarten (keine Verbreitungskarten) erstellt werden. Karte 2 zeigt das Vorkommen des Idioms gegen den Strom schwimmen: Für alle rot markierten Sprachen wurde in den Fragebögen ein in der lexikalischen Struktur sehr ähnliches und in der Bedeutung identisches Idiom eingetragen; in den blau markierten Sprachen existiert das Idiom den Angaben der Probanden/innen zufolge nicht. ${ }^{22}$ Eine Zusammenschau der Karten würde sodann eine raumbezogene Interpretation der Daten ermöglichen, vergleichbar der Ermittlung von Isoglossen in der Dialektgeographie oder von "isopleths" in der Typologieforschung.

Zugleich ist das WI-Projekt mit kulturellen Phänomenen der Phraseologie verbunden. Eine Präsentation der WIs in Form eines Lexikons bietet sich an. Vier Einheiten sollen konstant zu jedem Artikel ausgearbeitet werden:

(i) Die Idiome aller Sprachen werden mit exakten Beschreibungen der wörtlichen und figurativen Bedeutungen präsentiert. Das Beispiel der Karte 2 ist bis auf acht europäische in allen beteiligten Sprachen, auch in den ostasiatischen Kontrastsprachen, den Kleinsprachen und Dialekten, vertreten.

(ii) Sodann werden lexikalische und morphosyntaktische Besonderheiten der Idiome betrachtet. Für gegen den Strom schwimmen sind nur wenige Sonderwege zu verzeichnen: gleichzeitige Varianten "gegen den Wasserfall schwimmen" im Irischen und "gegen die Menschenmenge schwimmen" im Albanischen. In mehreren Sprachen sind Verben für "rudern" oder "laufen, gehen" statt "schwimmen" bekannt, wobei "Strom" und "Strömung" variieren können. Die Idiome verfügen offensichtlich über eine gemeinsame konzeptuelle Basis der gleichartigen figurativen Bedeutungen.

(iii) Ferner werden Spezifika der aktuellen Idiombedeutungen analysiert, wobei auf mögliche Unterschiede zu achten ist: Das Idiom einer Einzelsprache kann im Verlauf seiner Geschichte eine semantische Sonderentwicklung vollziehen, indem bestimmte relevante Merkmale des Zielkonzepts in den Vordergrund gerückt, andere unterdrückt werden, so dass sich das assoziative Umfeld verändern kann. Auch müssten Abweichungen diasystematischer Merkmale, textuellen, syntaktischen und stilistischen Verhaltens vermerkt werden. ${ }^{23}$

(iv) Der letzte Abschnitt bietet Raum für interpretierende Analysen der Daten. Aufgrund der relativ konstanten lexikalischen Struktur des Beispiels können keine Aussagen über die Ursachen und Wege der Verbreitung, über Entlehnungen und Gebersprachen gemacht werden (wie dies bei anderen WIs z. T. möglich ist). Zu berücksichtigen ist seine biblische Herkunft, damit die Möglichkeit, dass unterschiedliche Sprachen unabhängig von einander aus der

\footnotetext{
${ }^{22}$ Die Auflösung der Siglen der Sprachen findet sich am Ende des Beitrags.

${ }^{23}$ Bisher gibt es keine einheitliche Metasprache, mit Hilfe derer sich die Semantik von Idiomen umfassend und eindeutig beschreiben ließe.
} 
gleichen Quelle geschöpft haben, aber auch die "Unauffälligkeit" des Bildes, die nicht auf spezifisch biblischen Inhalten beruht.

\section{Erste Ergebnisse}

Als wichtigstes Ergebnis ist die Existenz sehr weit verbreiteter Idiome festzuhalten, was in der Phraseologieforschung in dieser Deutlichkeit bisher nicht bekannt war: Als "auffallend" galt das Vorkommen eines Idioms in drei, vier oder fünf Sprachen. Nach den Ergebnissen der WI-Umfragen hat es als auffallend zu gelten, wenn das Idiom in einer Sprache "nicht" vorkommt (vgl. das Beispiel Krokodilstränen weinen/vergießen in Abschnitt 6). Die meisten der abgefragten 100 Idiome geben ein ähnliches Bild ab wie das der Karte 2. Aufgrund der semasiologisch angelegten Abfrage der Idiome sind Strukturierungen des Raumes Europa nicht zu erwarten. Erst durch eine onomasiologisch angelegte Umfrage, die allerdings für Phraseologismen kaum durchzuführen ist, ${ }^{24}$ könnte sich eine areale Heteronymie abzeichnen. In wenigen Einzelfällen gewährt das Material der zurückgeschickten Fragebögen einen Einblick in eine Raumgliederung. Hier sei das Beispiel des in vielen Sprachen verbreiteten Idioms mit jmdm./etwas auf dem Kriegsfuß stehen genannt, das von norwegisch vare på krigstien med noen/noe bis spanisch estar en pie de guerra con alg./algo und von finnisch olla sotajalalla jkn kanssa bis tschechisch být s někým na válečné noze oder kroatisch biti s kim/čim na ratnoj nozi reicht. In diesen massiven Block großer Teile Europas ragt ein kleines südöstliches Areal hinein, in dem dieses Idiom nicht bekannt ist: Statt seiner wurde das quasiäquivalente Idiom der wörtlichen Lesart "(mit) jmdm. mit/auf/in Messer(n) sein" mehrfach notiert: z. B. bulgarisch на нож сьм с някого "auf Messer mit jmdm. sein", griechisch вí $\sigma \tau \alpha \mu \alpha \chi \alpha i \rho \iota \alpha \mu \varepsilon \kappa \alpha ́ \pi o \imath o v ~ " m i t ~ j m d m$. in den Messern sein", türkisch biriyle kanlı bıçaklı olmak "mit jmdm. blutig mit Messer sein".

Die meisten Ergebnisse des Projekts sind kultureller Art. Gemeinsamkeiten der Phraseologien europäischer Sprachen werden oft dem so genannten "gemeinsamen Kulturerbe" zugeschrieben, unter dem kulturelle Traditionen von der griechischen Antike, von Christentum, biblischem und mittelalterlichem lateinischen Schrifttum über Renaissance, Humanismus und Aufklärung bis hin zur klassischen Literatur subsumiert werden. Aufgrund der WI-Umfragen kann der recht vage Begriff des "kulturellen Erbes" als Ursache der phraseologischen Uniformität bereits ein wenig präzisiert werden. Erwartungsgemäß machen Idiome, die auf eine identifizierbare textuelle Quelle zurückgehen (vor allem Bibel und Fabeln), den größten Anteil der WIs aus. ${ }^{25}$ Anders ist es um die "klassische Literatur", d. h. um Dichtungen der Weltliteratur als postulierte Quellen von WIs, bestellt. Als bisher einziges weit verbreitetes Idiom dieser Art konnte gegen Windmühlen kämpfen, eine Anspielung auf Cervantes Roman "Don Quixote" (1605), ermittelt werden.

\footnotetext{
${ }^{24}$ Die Frage müsste z. B. lauten: "Welches Idiom gibt es in Ihrer Sprache für 'xy'?" - hier müsste eine eindeutige Bedeutungsdefinition gegeben werden.

${ }^{25}$ Vgl. die Typologie der Idiomen zugrunde liegenden kulturellen Phänomene in Dobrovol'skij/Piirainen (2005): 214ff).
} 
Neu ist ferner die Erkenntnis, dass neben das "europäische Kulturerbe antiker und christlicher Provenienz" ganz andere Textdomänen als Ursprung von WIs zu stellen sind. Eine wichtige Quelle bilden z. B. Vorstellungen von der Lebensweise nordamerikanischer Indianer, wie sie den Europäern durch die Erzählungen J. F. Coopers (1789-1863) vermittelt wurden und durch Filmproduktionen bis heute lebendig bleiben. Für eine Reihe von Idiomen dieser Herkunft (mit jmdm. die Friedenspfeife rauchen; der letzte der Mohikaner; mit jmdm./etw. auf dem Kriegsfuß stehen oder das Kriegsbeil ausgraben/begraben) wurde eine Verbreitung in vielen europäischen Sprachen nachgewiesen. Doch müssen derartige Beobachtungen anhand größerer empirischer Daten weiter verfolgt werden. 
Karte 1: etwas für lau tun/haben wollen

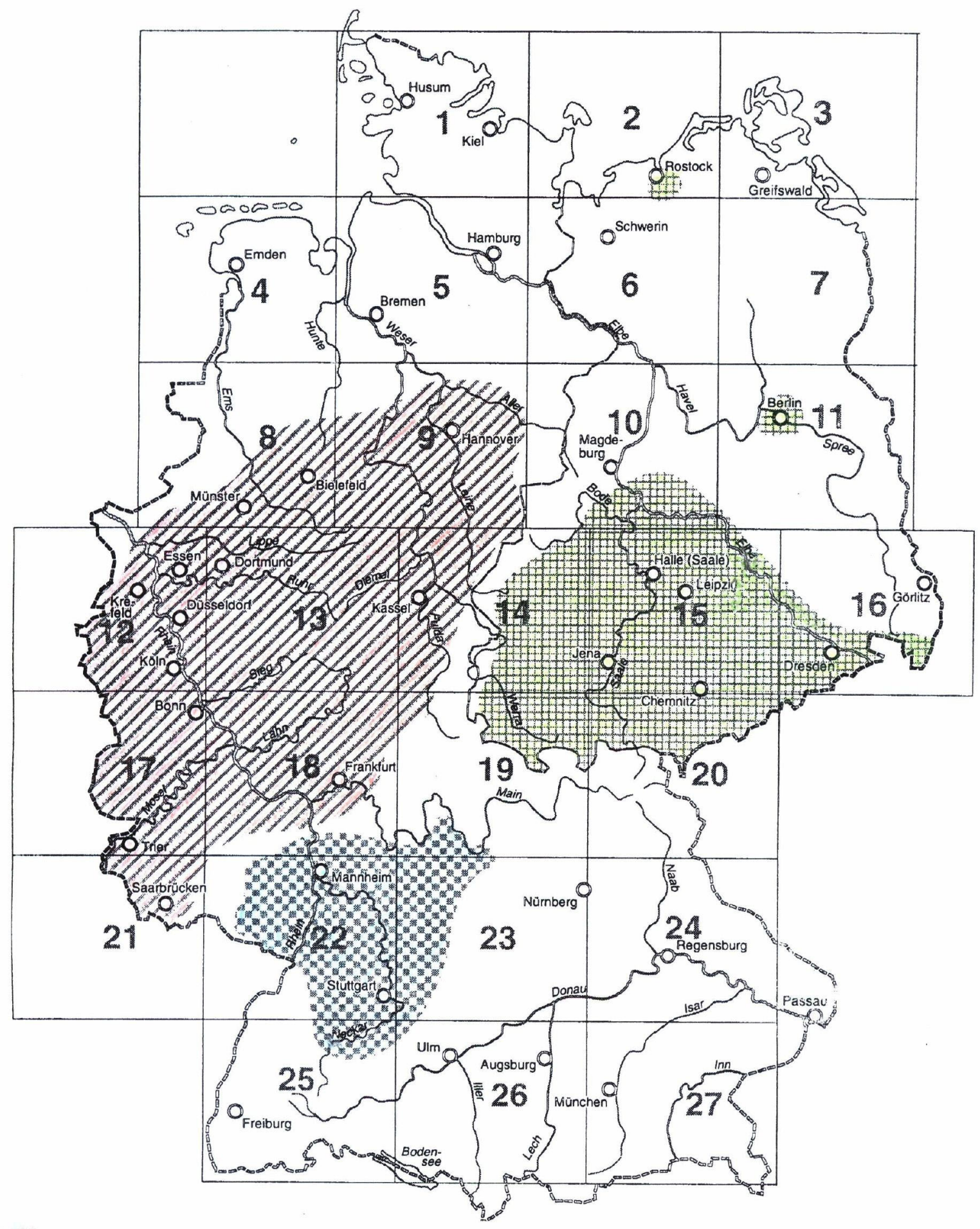


Karte 2: gegen den Strom schwimmen

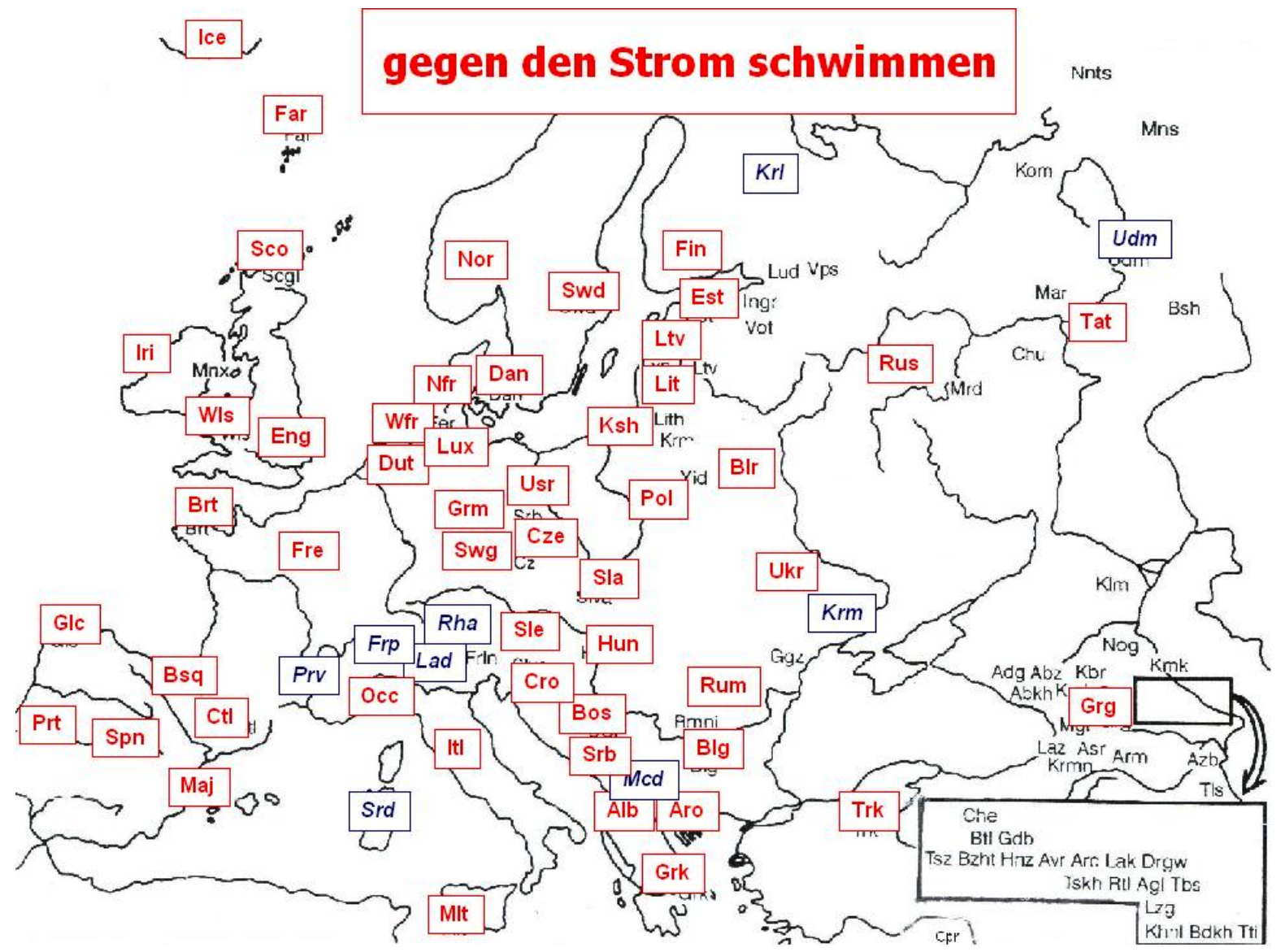

Siglen der Sprachen auf Karte 2

Siglen der Sprachen: rot: das Idiom existiert in der Sprache; blau: das Idiom wurde in der Sprache nicht nachgewiesen

$\begin{array}{llllll}\text { Alb } & \text { Albanisch } & \text { Grk } & \text { Griechisch } & \text { Prv } & \text { Provenzalisch } \\ \text { Aro } & \text { Aromanisch } & \text { Grm } & \text { Deutsch } & \text { Rha } & \text { Rätoromanisch } \\ \text { Blg } & \text { Bulgarisch } & \text { Hun } & \text { Ungarisch } & \text { Rum } & \text { Rumänisch } \\ \text { Blr } & \text { Weißrussisch } & \text { Ice } & \text { Isländisch } & \text { Rus } & \text { Russisch } \\ \text { Bos } & \text { Bosnisch } & \text { Iri } & \text { Irisch } & \text { Scg } & \text { Schottisch-Gälisch } \\ \text { Brt } & \text { Bretonisch } & \text { Itl } & \text { Italienisch } & \text { Sco } & \text { Schottisch/Scots } \\ \text { Bsq } & \text { Baskisch } & \text { Krl } & \text { Karelisch } & \text { Sla } & \text { Slowakisch } \\ \text { Cro } & \text { Kroatisch } & \text { Ksh } & \text { Kaschubisch } & \text { Sle } & \text { Slowenisch } \\ \text { Ctl } & \text { Katalanisch } & \text { Lad } & \text { Ladinisch } & \text { Spn } & \text { Spanisch } \\ \text { Cze } & \text { Tschechisch } & \text { Lit } & \text { Litauisch } & \text { Srd } & \text { Sardisch } \\ \text { Dan } & \text { Dänisch } & \text { Ltv } & \text { Lettisch } & \text { Swd } & \text { Schwedisch } \\ \text { Dut } & \text { Niederländisch } & \text { Lux } & \text { Lëtzebuergesch } & \text { Swg } & \text { Schweizerdeutsch } \\ \text { Eng } & \text { Englisch } & \text { Mal } & \text { Mallorquinisch } & \text { Tat } & \text { Tatarisch } \\ \text { Est } & \text { Estnisch } & \text { Mcd } & \text { Mazedonisch } & \text { Trk } & \text { Türkisch } \\ \text { Far } & \text { Färingisch } & \text { Mlt } & \text { Maltesisch } & \text { Udm } & \text { Udmurtisch } \\ \text { Fin } & \text { Finnisch } & \text { Nfr } & \text { Nordfriesisch } & \text { Ukr } & \text { Ukrainisch } \\ \text { Fre } & \text { Französisch } & \text { Nor } & \text { Norwegisch } & \text { Usr } & \text { Obersorbisch } \\ \text { Frp } & \text { Frankoprovenzalisch } & \text { Occ } & \text { Occitanisch } & \text { Wfr } & \text { Westfriesisch } \\ \text { Glc } & \text { Galicisch } & \text { Pol } & \text { Polnisch } & \text { Wls } & \text { Kymrisch } \\ \text { Grg } & \text { Georgisch } & \text { Prt } & \text { Portugiesisch } & & \end{array}$

